PROCEEDINGS OF THE

AMERICAN MATHEMATICAL SOCIETY

Volume 131, Number 5, Pages 1557-1564

S 0002-9939(02)06787-4

Article electronically published on October 1, 2002

\title{
COUNTABLY GENERATED HILBERT MODULES, THE KASPAROV STABILISATION THEOREM, AND FRAMES IN HILBERT MODULES
}

\author{
IAIN RAEBURN AND SHAUN J. THOMPSON
}

(Communicated by David R. Larson)

\begin{abstract}
We consider a class of countably generated Hilbert modules in which the generators are multipliers of the module, and prove a version of the Kasparov Stabilisation Theorem for these modules. We then extend recent work of Frank and Larson on frames in Hilbert modules.
\end{abstract}

The Kasparov Stabilisation Theorem says that every countably generated Hilbert module over a $C^{*}$-algebra $A$ is isomorphic to a direct summand of $l^{2}(A)=A_{A} \otimes$ $l_{\mathbb{C}}^{2}$. In applications, one often has to assume that the $C^{*}$-algebra $A$ is $\sigma$-unital, otherwise modules such as $A_{A}$ and $l^{2}(A)_{A}$ and their submodules are not countably generated. Here we propose a more general notion of countably generated module in which the generators can be multipliers of the module, and prove a version of Kasparov's theorem for these modules. With our definition, $A_{A}$ and $l^{2}(A)$ are countably generated for any $A$, and hence applications of our theorem should not require hypotheses on $A$.

We became interested in these ideas while studying recent work of Frank and Larson on frames in Hilbert modules, which are, loosely speaking, sequences for which one has a Parseval Identity and a reconstruction formula (see [5, 6]). The Kasparov Stabilisation Theorem is used in [5] to prove that every countably generated Hilbert module over a unital $C^{*}$-algebra admits frames; with our version of Kasparov's theorem, we can see that every countably generated Hilbert module admits a frame of multipliers. We can then extend many of Frank and Larson's results to frames of multipliers in countably generated modules over arbitrary $C^{*}$-algebras.

Multipliers of Hilbert modules have been used implicitly for many years (in [1, for example), and multiplier bimodules of imprimitivity bimodules were explicitly studied in [4. We review their properties in $\S 1$. In $\S 2$, we prove our version of Kasparov's theorem, following the argument of Mingo and Phillips (9], see also [8, 10]); the properties of multipliers considered in $\S 1$ turn out to be exactly what is needed to make their proof work. We discuss frames in $\S 3$. We consider only the standard normalised frames which generalise orthonormal bases, and prove the reconstruction formula for such frames (Theorem 3.4); again, with appropriate interpretation to accommodate multipliers, we can adapt arguments from [5]. The main tool is the frame transform (Theorem [3.5), which shows how a frame for a

Received by the editors February 16, 2001 and, in revised form, January 3, 2002.

2000 Mathematics Subject Classification. Primary 46L08.

This research was supported by the Australian Research Council. 
Hilbert module $\mathcal{H}_{A}$ gives an embedding of $\mathcal{H}_{A}$ as a submodule of $l^{2}(A)$. We close by briefly discussing other implications of our theorem, which include a new version of the Brown-Green-Rieffel Theorem for non- $\sigma$-unital $C^{*}$-algebras.

Conventions. We use the theory of Hilbert modules as discussed in [8] and [10], and in particular we use right modules throughout. If $\mathcal{H}$ and $\mathcal{K}$ are Hilbert modules over the same $C^{*}$-algebra $A$, we denote by $\mathcal{L}\left(\mathcal{H}_{A}, \mathcal{K}_{A}\right)$ or $\mathcal{L}(\mathcal{H}, \mathcal{K})$ the Banach space of adjointable operators from $\mathcal{H}$ to $\mathcal{K}$. We denote by $\Theta_{k, h}$ the rank-one operator $g \mapsto k \cdot\langle h, g\rangle_{A}$, and by $\mathcal{K}\left(\mathcal{H}_{A}, \mathcal{K}_{A}\right)$ the closed span of these operators in $\mathcal{L}\left(\mathcal{H}_{A}, \mathcal{K}_{A}\right)$. We write $l^{2}(A)$ for the Hilbert $A$-module of sequences $\left(a_{n}\right)$ in $A$ such that $\sum a_{n}^{*} a_{n}$ converges in $A$, with inner product given by $\left\langle\left(a_{n}\right),\left(b_{n}\right)\right\rangle_{A}:=\sum a_{n}^{*} b_{n}$; this module is discussed in [10, §2.1], for example.

\section{Multiplier modules}

To motivate the next definition, recall that we can identify the multiplier algebra $M(A)$ with the algebra $\mathcal{L}\left(A_{A}\right)$ of adjointable operators on the trivial module $A_{A}$. For those used to the double-centraliser definition of $M(A)$, the identification takes $m \in M(A)$ to the left centraliser $L_{m}: a \mapsto m a$; the existence of the adjoint is equivalent to the existence of the right centraliser. Now for a Hilbert $A$-module $\mathcal{H}$, we define $M(\mathcal{H}):=\mathcal{L}\left(A_{A}, \mathcal{H}_{A}\right)$.

Remark 1.1. It is crucial in defining $M(\mathcal{H})$ that we know which algebra $A$ is the coefficient algebra: given $\mathcal{H}_{A}$, we can view $\mathcal{H}$ as a Hilbert module over any $C^{*}$ subalgebra of $M(A)$ containing the range of the inner product, but the multiplier bimodule will change. For example, $M\left(A_{A}\right)=\mathcal{L}(A, A)=M(A)$, whereas $M\left(A_{M(A)}\right)=\mathcal{L}(M(A), A)=A$.

Proposition 1.2. The set $M(\mathcal{H})$ is a Hilbert $M(A)$-module with $(m \cdot b)(a):=m(b a)$ and $\langle m, n\rangle_{M(A)}:=m^{*} \circ n$ for $m, n \in M(\mathcal{H})$ and $a \in M(A)$. For $h \in \mathcal{H}, \iota_{\mathcal{H}}(h)$ : $a \mapsto h \cdot a$ is adjointable with adjoint $\iota_{\mathcal{H}}(h)^{*}: k \mapsto\langle h, k\rangle_{A}$, and the map $\iota_{\mathcal{H}}$ embeds $\mathcal{H}$ as a closed $M(A)$-submodule of $M(\mathcal{H})$. We call $M(\mathcal{H})_{M(A)}$ the multiplier module of $\mathcal{H}_{A}$.

Proof. Each $m^{*} \circ n$ is an adjointable operator on $A_{A}$, and hence belongs to $M(A)=$ $\mathcal{L}\left(A_{A}\right)$; it is a routine exercise to verify that $M(\mathcal{H})$ is an inner-product $M(A)$ module. To establish completeness of $M(\mathcal{H})$ we use the following lemma, which follows from a standard linking-algebra argument.

Lemma 1.3. Let $\mathcal{H}$ and $\mathcal{K}$ be Hilbert $A$-modules and $T \in \mathcal{L}(\mathcal{H}, \mathcal{K})$ be an adjointable operator. Then $\left\|T^{*} \circ T\right\|_{\mathcal{L}(\mathcal{H})}=\|T\|_{\mathcal{L}(\mathcal{H}, \mathcal{K})}^{2}$.

We know that $M(\mathcal{H})=\mathcal{L}(A, \mathcal{H})$ is complete in the operator norm, and the lemma says that this agrees with the norm induced by the inner product. Thus $M(\mathcal{H})$ is a Hilbert $M(A)$-module.

The calculation

$$
\left\langle\iota_{\mathcal{H}}(h)(a), k\right\rangle_{A}=\langle h \cdot a, k\rangle_{A}=a^{*}\langle h, k\rangle_{A}=\left\langle a,\langle h, k\rangle_{A}\right\rangle_{A}
$$

shows that $k \mapsto\langle h, k\rangle_{A}$ is an adjoint for $\iota_{\mathcal{H}}(h)$. To see that $\iota_{\mathcal{H}}: \mathcal{H} \rightarrow M(\mathcal{H})$ is an $M(A)$-module homomorphism, it suffices to show that it is an $A$-module homomorphism. But then for $h \in \mathcal{H}$ and $a, b \in A$ we have

$$
\left(\iota_{\mathcal{H}}(h) \cdot b\right)(a)=\iota_{\mathcal{H}}(h)(b a)=h \cdot(b a)=(h \cdot b) \cdot a=\iota_{\mathcal{H}}(h \cdot b)(a),
$$


and hence $\iota_{\mathcal{H}}(h) \cdot b=\iota_{\mathcal{H}}(h \cdot b)$. Another routine calculation shows that $\iota_{\mathcal{H}}$ respects the inner products; this implies that $\iota_{\mathcal{H}}$ is isometric and hence has closed range.

Just as we usually identify $A$ with its canonical image in $M(A)$, we usually suppress the canonical map $\iota_{\mathcal{H}}$, and think of $\mathcal{H}$ as a submodule of $M(\mathcal{H})$. Then we have:

Proposition 1.4. Let $\mathcal{H}$ be a Hilbert $A$-module and suppose $m \in M(\mathcal{H})$. Then $m \cdot a=m(a)$ belongs to $\mathcal{H}$ for every $a \in A$, and $\langle m, h\rangle_{M(A)}=m^{*}(h)$ belongs to $A$ for every $h \in \mathcal{H}$.

Proof. Strictly speaking, these statements involve the map $\iota_{\mathcal{H}}$. For example, the first statement says $m \cdot a=\iota_{\mathcal{H}}(m(a))$. To verify it, let $b \in A$. Then $(m \cdot a)(b)=$ $m(a b)=m(a) \cdot b=\iota_{\mathcal{H}}(m(a))(b)$, as required. Similar arguments give the second statement.

\section{The Kasparov Stabilisation Theorem}

Definition 2.1. A Hilbert $A$-module $\mathcal{H}$ is countably generated in $M(\mathcal{H})$ if there is a sequence $\left\{h_{i}\right\} \subseteq M(\mathcal{H})$ such that the elements $h_{i} \cdot a$ span a dense submodule of $\mathcal{H}$.

Example 2.2. The Hilbert $A$-module $l^{2}(A)$ is countably generated in $M\left(l^{2}(A)\right)$ for any $C^{*}$-algebra $A$. To see this, let $i \in \mathbb{N}$, and define $e_{i}: A \rightarrow l^{2}(A)$ by $e_{i}(a):=(0,0, \ldots, 0, a, 0, \ldots)$; then $e_{i}$ is adjointable with adjoint $e_{i}^{*}:\left(a_{n}\right) \mapsto a_{i}$, and hence $e_{i} \in M\left(l^{2}(A)\right)$. We claim that for $h \in l^{2}(A)$,

$$
h=\sum_{i=1}^{\infty} e_{i} \cdot\left\langle e_{i}, h\right\rangle_{M(A)}
$$

in the sense that the infinite sum converges in $l^{2}(A)$ to $h$; in particular, because we know from Proposition 1.4 that $\left\langle e_{i}, h\right\rangle_{M(A)} \in A$, this implies that the $\left\{e_{i}\right\}$ generate $l^{2}(A)$. Suppose $h=\left(a_{n}\right)$. By Proposition 1.4 we have $\left\langle e_{i}, h\right\rangle_{M(A)}=e_{i}^{*}(h)=a_{i}$, and hence $e_{i} \cdot\left\langle e_{i}, h\right\rangle_{M(A)}=e_{i}\left(a_{i}\right)$. Thus $\sum_{i=1}^{n} e_{i} \cdot\left\langle e_{i}, h\right\rangle_{M(A)}=\left(a_{1}, \ldots, a_{n}, 0, \ldots\right)$, and

$$
\left\|h-\sum_{i=1}^{n} e_{i} \cdot\left\langle e_{i}, h\right\rangle_{M(A)}\right\|^{2}=\left\|\left(0, \ldots, 0, a_{n+1}, a_{n+2}, \ldots\right)\right\|^{2}=\left\|\sum_{j=n+1}^{\infty} a_{j}^{*} a_{j}\right\|,
$$

which converges to 0 as $n \rightarrow \infty$ because $h=\left(a_{n}\right) \in l^{2}(A)$.

Theorem 2.3 (The Kasparov Stabilisation Theorem). Suppose that $\mathcal{H}$ is a Hilbert $A$-module which is countably generated in $M(\mathcal{H})$. Then $l^{2}(A)$ is isomorphic as a Hilbert A-module to $\mathcal{H} \oplus l^{2}(A)$.

Following the argument in [10, $\S 5.5]$, we let $\left\{m_{i}\right\} \subseteq M(\mathcal{H})$ be a generating sequence for $\mathcal{H}$, normalised so that $\left\|m_{i}\right\|=1$ for each $i$. Let $\left\{x_{i}\right\} \subseteq M(\mathcal{H})$ be a sequence obtained by listing the generators $m_{i}$ in such a way that each appears infinitely often. Let $\left\{e_{i}\right\}$ be the generating set for $l^{2}(A)$ described in Example 2.2 We identify $\mathcal{H}$ and $l^{2}(A)$ with their images in $\mathcal{H} \oplus l^{2}(A)$.

The next claim in [10] is that

$$
T:=\sum_{n=1}^{\infty} 2^{-n} \Theta_{x_{n}, e_{n}}+4^{-n} \Theta_{e_{n}, e_{n}}
$$


converges in norm to an operator $T$ in $\mathcal{K}\left(l^{2}(A), \mathcal{H} \oplus l^{2}(A)\right)$. There is a potential problem here, since $\Theta_{x_{n}, e_{n}}$, for example, is defined to be an operator from $M\left(l^{2}(A)\right)$ to $M(\mathcal{H})$. However, the multiplier-like properties described in Proposition [1.4 are exactly what we need to see that $\Theta_{x_{n}, e_{n}}$ also maps $l^{2}(A)$ to $\mathcal{H}$ :

Lemma 2.4. Suppose $\mathcal{H}$ and $\mathcal{K}$ are Hilbert $A$-modules, and let $m \in M(\mathcal{H}), n \in$ $M(\mathcal{K})$. Then the formula

$$
\Theta_{n, m}(h)=n \cdot\langle m, h\rangle_{M(A)}
$$

defines an adjointable map $\Theta_{n, m}: \mathcal{H} \rightarrow \mathcal{K}$ with adjoint $\Theta_{m, n}$, and $\left\|\Theta_{n, m}\right\| \leq$ $\|m\|\|n\|$.

Proof. For $h \in \mathcal{H}$, Proposition 1.4 tells us that $\langle m, h\rangle_{M(A)} \in A$ and $n \cdot\langle m, h\rangle_{M(A)} \in$ $\mathcal{K}$. For $h \in \mathcal{H}, k \in \mathcal{K}$, we have

$$
\begin{aligned}
\left\langle\Theta_{n, m}(h), k\right\rangle_{A} & =\left\langle n \cdot\langle m, h\rangle_{M(A)}, k\right\rangle_{A}=\langle m, h\rangle_{M(A)}^{*}\langle n, k\rangle_{M(A)} \\
& =\langle h, m\rangle_{M(A)}\langle n, k\rangle_{M(A)}=\left\langle h, m \cdot\langle n, k\rangle_{M(A)}\right\rangle_{A} \\
& =\left\langle h, \Theta_{m, n}(k)\right\rangle_{A},
\end{aligned}
$$

so $\Theta_{m, n}$ is an adjoint for $\Theta_{n, m}$. The estimate for $\left\|\Theta_{n, m}\right\|$ is easy.

Proof of Theorem 2.3. We now set $S_{n}=\sum_{k=1}^{n} 2^{-k} \Theta_{x_{k}, e_{k}}+4^{-k} \Theta_{e_{k}, e_{k}}$. Then the estimate in Lemma 2.4 shows that

$$
\left\|S_{m}-S_{n}\right\|=\left\|\sum_{k=n+1}^{m} 2^{-k} \Theta_{x_{k}, e_{k}}+4^{-k} \Theta_{e_{k}, e_{k}}\right\| \leq \sum_{k=n+1}^{m} 2^{-k}+4^{-k},
$$

which converges to 0 as $m, n \rightarrow \infty$. So the series $\sum_{k=1}^{\infty} 2^{-k} \Theta_{x_{k}, e_{k}}+4^{-k} \Theta_{e_{k}, e_{k}}$ does still converge, though the sum $T \in \mathcal{L}\left(l^{2}(A), \mathcal{H} \oplus l^{2}(A)\right)$ is now an adjointable operator rather than a compact one.

Since $\left\langle e_{n}, e_{m}\right\rangle_{M(A)}=\delta_{m, n} 1_{M(A)}$, we have $T\left(e_{m} \cdot a\right)=2^{-m}\left(x_{m} \cdot a\right)+4^{-m}\left(e_{m} \cdot a\right)$, and $T\left(2^{m}\left(e_{m} \cdot a\right)\right)=\left(x_{m} \cdot a\right)+2^{-m}\left(e_{m} \cdot a\right)$. Thus for all $n \in \mathbb{N}$ satisfying $x_{n}=x_{m}$, we have $T\left(2^{n}\left(e_{n} \cdot a\right)\right)=\left(x_{m} \cdot a\right)+2^{-n}\left(e_{n} \cdot a\right)$, or, equivalently,

$$
x_{m} \cdot a=T\left(2^{n}\left(e_{n} \cdot a\right)\right)-2^{-n}\left(e_{n} \cdot a\right) .
$$

But there are infinitely many $n \in \mathbb{N}$ with $x_{n}=x_{m}$, so (2.3) holds for infinitely many $n \in \mathbb{N}$. Since $2^{-n}\left(e_{n} \cdot a\right) \rightarrow 0$ in $l^{2}(A)$, it follows that $x_{m} \cdot a \in \overline{T\left(l^{2}(A)\right)}$. What is more, $T\left(4^{m}\left(e_{m} \cdot a\right)\right)=2^{m}\left(x_{m} \cdot a\right)+e_{m} \cdot a$, so $e_{m} \cdot a=T\left(4^{m}\left(e_{m} \cdot a\right)\right)-2^{m}\left(x_{m} \cdot a\right)$ also belongs to $\overline{T\left(l^{2}(A)\right)}$. Since the elements $x_{m} \cdot a$ span $\mathcal{H}$, and $e_{m} \cdot a$ span $l^{2}(A)$, it follows that $\mathcal{H} \oplus l^{2}(A)=\overline{T\left(l^{2}(A)\right)}$.

The series $\sum_{n=1}^{\infty} 2^{-n} \Theta_{e_{n}, x_{n}}+4^{-n} \Theta_{e_{n}, e_{n}}$ converges in norm to the adjoint $T^{*}$ of $T$, and similar arguments show that $T^{*}\left(4^{m}\left(e_{m} \cdot a\right)\right)=e_{m} \cdot a$ for fixed $a \in A, m \in \mathbb{N}$. Thus $l^{2}(A)=\overline{T^{*}\left(\mathcal{H} \oplus l^{2}(A)\right)}$. By polar decomposing $T$ we obtain a Hilbert-module isomorphism of $l^{2}(A)$ onto $\mathcal{H} \oplus l^{2}(A)$ (see, for example, [10, Lemma 2.34]).

\section{Frames in Hilbert modules}

Definition 3.1. Let $A$ be a $C^{*}$-algebra and $\mathcal{H}$ a Hilbert $A$-module. A sequence $\left\{h_{i}\right\} \subseteq M(\mathcal{H})$ is a standard normalised frame for $\mathcal{H}$ in $M(\mathcal{H})$ if

$$
\langle h, h\rangle_{A}=\sum_{i=1}^{\infty}\left\langle h, h_{i}\right\rangle_{M(A)}\left\langle h_{i}, h\right\rangle_{M(A)} \quad \text { for every } h \in \mathcal{H},
$$

in the sense that the series converges in norm with sum $\langle h, h\rangle_{A}$. 
If $A$ is unital, then $M(\mathcal{H})=\mathcal{H}$, this definition coincides with that in [5], and the results of this section are all due to Frank and Larson. However, allowing $h_{i}$ to be in $M(\mathcal{H})$ rather than $\mathcal{H}$ is definitely more general:

Example 3.2. For any $C^{*}$-algebra $A$, the set $\left\{e_{1}, e_{2}, \ldots\right\}$ is a standard normalised frame for $l^{2}(A)$. To see this, let $h=\left(a_{n}\right) \in l^{2}(A)$. Then $\left\langle e_{i}, h\right\rangle_{M(A)}=a_{i}$, so

$$
\sum_{i=1}^{n}\left\langle h, e_{i}\right\rangle_{M(A)}\left\langle e_{i}, h\right\rangle_{M(A)}=\sum_{i=1}^{n} a_{i}^{*} a_{i}
$$

this series converges in $A$ because $h=\left(a_{n}\right)$ belongs to $l^{2}(A)$, and the limit is $\langle h, h\rangle_{A}$ by definition of the inner product on $l^{2}(A)$.

Every orthonormal basis for a Hilbert space is a standard normalised frame; a main point of frame theory is that there are many more, including the image of any orthonormal basis under an orthogonal projection. Hilbert modules, on the other hand, do not usually have orthonormal bases. So it is quite remarkable that they always admit frames:

Corollary 3.3. If $A$ is a $C^{*}$-algebra and $\mathcal{H}$ is a Hilbert $A$-module which is countably generated in $M(\mathcal{H})$, then $\mathcal{H}$ has a standard normalised frame.

Before the proof, we make some general comments. Suppose $\mathcal{M}$ is a closed submodule of a Hilbert module $\mathcal{H}$. We say that $\mathcal{M}$ is a complemented submodule of $\mathcal{H}$ if the map $\Sigma_{\mathcal{M}}:(m, n) \mapsto m+n$ is a Hilbert module isomorphism of $\mathcal{M} \oplus \mathcal{M}^{\perp}$ onto $\mathcal{H}$. If so, there is an orthogonal projection $P: \mathcal{H} \rightarrow \mathcal{M}$ characterised by the property that for $h \in \mathcal{H}, P h$ is the unique element of $\mathcal{M}$ such that $h-P h$ is in $\mathcal{M}^{\perp}$. Just as for Hilbert spaces, and by essentially the same proof, the orthogonal projections onto complemented submodules can be characterised as the adjointable operators $P \in \mathcal{L}(\mathcal{H})$ satisfying $P^{2}=P=P^{*}$. If $\left\{e_{i}\right\}$ is a standard normalised frame in the usual sense, then $\left\{P\left(e_{i}\right)\right\}$ is a standard normalised frame in $P \mathcal{H}$. Here we note that $P \circ e_{i}$ belongs to $M(P \mathcal{H})=\mathcal{L}\left(A_{A}, P \mathcal{H}\right)$ for each $i$, and $\left\{P \circ e_{i}\right\}$ is then a standard normalised frame for $P \mathcal{H}$ in $M(P \mathcal{H})$.

Proof of Corollary 3.3 Theorem 2.3 says there is an isomorphism $T$ of $\mathcal{H}$ onto a complemented submodule $\mathcal{M}$ of $l^{2}(A)$. Let $P: l^{2}(A) \rightarrow \mathcal{M}$ be the orthogonal projection of $l^{2}(A)$ onto $\mathcal{M}$. Then $\left\{T^{-1} \circ P \circ e_{i}\right\}$ is a standard normalised frame for $\mathcal{H}$ in $M(\mathcal{H})$.

Theorem 3.4 (The reconstruction formula). Let $\mathcal{H}$ be a Hilbert A-module which is countably generated in $M(\mathcal{H})$. Suppose $\left\{h_{i}\right\}$ is a sequence in $M(\mathcal{H})$. A sequence $\left\{h_{i}\right\}$ in $M(\mathcal{H})$ is a standard normalised frame for $\mathcal{H}$ in $M(\mathcal{H})$ if and only if for each $h \in \mathcal{H}$ we have

$$
h=\sum_{i}^{\infty} h_{i} \cdot\left\langle h_{i}, h\right\rangle_{M(A)},
$$

in the sense that this sum converges in norm to $h$. 
Suppose first that $\left\{h_{i}\right\} \subseteq M(\mathcal{H})$ satisfies the reconstruction formula (3.2). Then

$$
\begin{aligned}
\langle h, h\rangle_{A} & =\left\langle h, \sum_{i=1}^{\infty} h_{i} \cdot\left\langle h_{i}, h\right\rangle_{M(A)}\right\rangle_{M(A)} \\
& =\left\langle h, \lim _{n \rightarrow \infty} \sum_{i=1}^{n} h_{i} \cdot\left\langle h_{i}, h\right\rangle_{M(A)}\right\rangle_{M(A)} \\
& =\lim _{n \rightarrow \infty} \sum_{i=1}^{n}\left\langle h, h_{i}\right\rangle_{M(A)}\left\langle h_{i}, h\right\rangle_{M(A)},
\end{aligned}
$$

so that $\left\{h_{i}\right\}$ is a standard normalised frame for $\mathcal{H}$.

For the converse, we use the following theorem which is of interest in its own right.

Theorem 3.5 (The frame transform). Let $\mathcal{H}$ be a Hilbert A-module which is countably generated in $M(\mathcal{H})$, and let $\left\{h_{i}\right\}$ be a standard normalised frame for $\mathcal{H}$ in $M(\mathcal{H})$. The frame transform for $\left\{h_{i}\right\}$ is the map $\theta: \mathcal{H} \rightarrow l^{2}(A)$ defined by $\theta(h)=\left(\left\langle h_{i}, h\right\rangle_{M(A)}\right)$. The frame transform preserves the inner product, and is adjointable with adjoint $\theta^{*}: l^{2}(A) \rightarrow \mathcal{H}$ satisfying $\theta^{*} \circ e_{i}=h_{i}$.

Proof. Notice that each $\left\langle h_{i}, h\right\rangle_{M(A)}$ belongs to $A$ because $h_{i}$ is a multiplier, and the sequence $\theta(h)$ belongs to $l^{2}(A)$ because $\left\{h_{i}\right\}$ is a standard normalised frame; the frame identity (3.1) and the usual polarisation identity show that $\theta$ preserves the inner product. As a first step in constructing $\theta^{*}$, we claim that there is a well-defined bounded linear map $S: \operatorname{span}\left\{e_{i} \cdot a: a \in A, i \in \mathbb{N}\right\} \rightarrow \mathcal{H}$ which satisfies $S\left(\sum_{i=1}^{n} e_{i} \cdot a_{i}\right)=\sum_{i=1}^{n} h_{i} \cdot a_{i}$. To see that $S$ is well-defined, suppose $\sum_{i=1}^{n} e_{i} \cdot a_{i}=\sum_{j=1}^{m} e_{j} \cdot b_{j}$. Then we have

$$
a_{k}=\left\langle e_{k}, \sum_{i=1}^{n} e_{i} \cdot a_{i}\right\rangle_{M(A)}=\left\langle e_{k}, \sum_{j=1}^{m} e_{j} \cdot b_{j}\right\rangle_{M(A)}=b_{k}
$$

which implies that $m=n$ and that $\sum_{i=1}^{n} h_{i} \cdot a_{i}=\sum_{j=1}^{n} h_{j} \cdot b_{j}$. Thus $S$ is welldefined.

Next, note that for $h \in \mathcal{H}, x=\sum_{i=1}^{n} e_{i} \cdot a_{i} \in \operatorname{span}\left\{e_{i} \cdot a: a \in A\right\}$, we have

$$
\begin{aligned}
\langle\theta h, x\rangle_{A} & =\left\langle\left(\left\langle h_{k}, h\right\rangle_{M(A)}\right), \sum_{i=1}^{n} e_{i} \cdot a_{i}\right\rangle_{A} \\
& =\sum_{i=1}^{n}\left\langle\left(\left\langle h_{k}, h\right\rangle_{M(A)}\right), e_{i}\right\rangle_{M(A)} a_{i}=\sum_{i=1}^{n}\left\langle h, h_{i}\right\rangle_{M(A)} a_{i} \\
& =\left\langle h, \sum_{i=1}^{n} h_{i} \cdot a_{i}\right\rangle_{A}=\langle h, S x\rangle_{A} .
\end{aligned}
$$

To see that $S$ is bounded, let $x=\sum_{i=1}^{n} e_{i} \cdot a_{i}$. Then, because $\theta$ preserves the inner product and hence the norm, we deduce from (3.3) that

$$
\|S(x)\|=\sup _{\|h\|=1}\left\|\langle S(x), h\rangle_{A}\right\|=\sup _{\|h\|=1}\left\|\langle x, \theta h\rangle_{A}\right\| \leq \sup _{\|h\|=1}\|x\|\|\theta h\|=\|x\| .
$$

Now, because $\operatorname{span}\left\{e_{i} \cdot a\right\}$ is dense in $l^{2}(A)$, there is a unique extension of $S$ to a bounded linear map $T: l^{2}(A) \rightarrow \mathcal{H}$. We have $T\left(\left(a_{i}\right)\right)=\sum_{i} h_{i} \cdot a_{i}$ for $\left(a_{i}\right) \in l^{2}(A)$ by continuity of $T$, and letting $n \rightarrow \infty$ in (3.3) shows that $T$ is an adjoint for $\theta$. 
To finish the proof of Theorem [3.4, let $\left\{h_{i}\right\} \subseteq M(\mathcal{H})$ be a standard normalised frame for $\mathcal{H}$, and let $\theta: \mathcal{H} \rightarrow l^{2}(A)$ be the frame transform. Since $\theta$ preserves the inner product, we have $\theta^{*} \circ \theta=1_{\mathcal{L}(\mathcal{H})}$. Then for $h \in \mathcal{H}$,

$$
\begin{aligned}
\left\|h-\sum_{i=1}^{n} h_{i} \cdot\left\langle h_{i}, h\right\rangle_{M(A)}\right\| & =\left\|h-\sum_{i=1}^{n} h_{i} \cdot h_{i}^{*}(h)\right\| \\
& =\left\|\theta^{*} \circ \theta(h)-\sum_{i=1}^{n}\left(\theta^{*} \circ e_{i}\right)\left(\left\langle h_{i}, h\right\rangle_{M(A)}\right)\right\| \\
& =\left\|\theta(h)-\sum_{i=1}^{n} e_{i} \cdot\left\langle h_{i}, h\right\rangle_{M(A)}\right\| \\
& =\left\|\theta(h)-\sum_{i=1}^{n} e_{i} \cdot\left\langle e_{i}, \theta(h)\right\rangle_{M(A)}\right\|
\end{aligned}
$$

which converges to zero by (2.2). This gives the reconstruction formula (3.2).

\section{Concluding Remarks}

4.1. The Brown-Green-Rieffel Theorem. The Brown-Green-Rieffel Theorem asserts that two $\sigma$-unital $C^{*}$-algebras $A$ and $B$ are stably isomorphic if and only if they are Morita equivalent, that is, if and only if there is an $A$ - $B$ imprimitivity bimodule (see [3, Theorem 1.2] or [10, Theorem 5.55]). In Blackadar's short proof of this theorem [2], the $\sigma$-unital hypothesis is only used to ensure that every $A$ - $B$ imprimitivity bimodule module $X$ is countably generated over both $A$ and $B$ (this follows from [10, Proposition 5.50] because $\mathcal{K}\left(X_{B}\right) \cong A$ and $\left.\mathcal{K}\left({ }_{A} X\right) \cong B\right)$ and that $l^{2}(B)$ is countably generated, so that he can apply the Kasparov Stabilisation Theorem. By following Blackadar's proof and using Theorem 2.3] in place of Kasparov's theorem, we can deduce that two $C^{*}$-algebras $A$ and $B$ are stably isomorphic if and only if there is an $A-B$ imprimitivity bimodule $X$ which is countably generated in $M(X)$ both as an $A$-module and as a $B$-module. (We can see by considering a non-separable Hilbert space that the hypothesis has to be imposed on both sides.) This version of the Brown-Green-Rieffel Theorem makes it clear that the countability hypotheses are intrinsic to the equivalence relations under consideration rather than the underlying $C^{*}$-algebras.

4.2. $K$-theory. Kasparov originally proved his stabilisation theorem as a tool for the construction of $K K$-theory, and it is often assumed in $K K$-theory that the $C^{*}$-algebras are $\sigma$-unital. At this stage, it does not seem likely that our version will allow all these hypotheses to be removed: they are also being used, for example, in the proof of Kasparov's Technical Theorem [7, Theorem 2.2.1].

4.3. Finitely generated modules. By saying that a Hilbert $A$-module $\mathcal{H}$ is finitely generated, one usually means that there are elements $\left\{h_{i}: 1 \leq i \leq n\right\}$ such that $\mathcal{H}=\left\{\sum_{i=1}^{n} h_{i} \cdot a_{i}: a_{i} \in A\right\}$ - in other words, no closure is taken. We can now say that $\mathcal{H}$ is finitely generated in $M(\mathcal{H})$ if we can find $h_{i} \in M(\mathcal{H})$ which do the same thing; the property $M(\mathcal{H}) \cdot A \subset \mathcal{H}$ ensures that the finite sums are in $\mathcal{H}$. With this definition, $A_{A}$ is always singly generated by $1_{M(A)}$, and $A^{n}$ is $n$-generated; this gives further evidence that we have hit on the right notion of generator. 
If a Hilbert $A$-module $\mathcal{H}$ is finitely generated in $M(\mathcal{H})$, we can follow the arguments of Frank and Larson to see that $\mathcal{H}$ is a complemented submodule of some $A^{n}$ (see [5, Theorem 5.9]).

\section{REFERENCES}

[1] S. Baaj and G. Skandalis, $C^{*}$-algèbres de Hopf et théorie de Kasparov équivariante, K-Theory 2 (1989), 683-721. MR 90j:46061

[2] B. Blackadar, article to appear in the Encyclopedia of Mathematics.

[3] L.G. Brown, P. Green and M.A. Rieffel, Stable isomorphism and strong Morita equivalence of $C^{*}$-algebras, Pacific J. Math. 71 (1977), 349-363. MR 57:3866

[4] S. Echterhoff and I. Raeburn, Multipliers of imprimitivity bimodules and Morita equivalence of crossed products, Math. Scand. 76 (1995), 289-309. MR 97h:46093

[5] M. Frank and D.R. Larson, Frames in Hilbert $C^{*}$-modules and $C^{*}$-algebras, J. Operator Theory, to appear.

[6] M. Frank and D.R. Larson, A module frame concept for Hilbert $C^{*}$-modules, The Functional and Harmonic Analysis of Wavelets and Frames, Contemp. Math. vol. 247, Amer. Math. Soc., Providence, 1999, pages 207-233. MR 2001b:46094

[7] K.K. Jensen and K. Thomsen, Elements of $K K$-Theory, Birkhäuser, Boston, 1991. MR 94b:19008

[8] E.C. Lance, Hilbert $C^{*}$-Modules: A Toolkit for Operator Algebraists, London Math. Soc. Lecture Note Series, vol. 210, Cambridge Univ. Press, 1994.

[9] J.A. Mingo and W.J. Phillips, Equivariant triviality theorems for Hilbert $C^{*}$-modules, Proc. Amer. Math. Soc. 91 (1984), 225-230. MR 85f:46111

[10] I. Raeburn and D.P. Williams, Morita Equivalence and Continuous-Trace $C^{*}$-Algebras, Math. Surveys and Monographs, vol. 60, Amer. Math. Soc., Providence, 1998. MR 2000c:46108

Department of Mathematics, University of Newcastle, New South Wales 2308, AusTRALIA

E-mail address: iain@frey.newcastle.edu.au

Department of Mathematics, University of Newcastle, New South Wales 2308, AusTRALIA

E-mail address: shaun@frey.newcastle.edu.au 